\title{
Cerebrospinal fluid biomarkers for differentiation of frontotemporal lobar degeneration from Alzheimer's disease
}

\author{
David J. Irwin ${ }^{1,2}$, John Q. Trojanowski ${ }^{1}$ and Murray Grossman ${ }^{2 *}$ \\ Department of Pathology and Laboratory Medicine, Center for Neurodegenerative Disease Research, Alzheimer's Disease Core Center, Institute on Aging, \\ University of Pennsylvania, Philadelphia, PA, USA \\ 2 Department of Neurology, Center for Frontotemporal Dementia, Perelman School of Medicine, University of Pennsylvania, Philadelphia, PA, USA
}

\author{
Edited by: \\ Manuel Menéndez-González, \\ Hospital Álvarez-Buylla, Spain \\ Reviewed by: \\ Junming Wang, University of \\ Mississippi Medical Center, USA \\ Angela de Iuliis, University of \\ Padova, Italy \\ *Correspondence: \\ Murray Grossman, Department of \\ Neurology, University of \\ Pennsylvania School of Medicine, \\ Hospital of the University of \\ Pennsylvania, 3400 Spruce Street, \\ Philadelphia, PA 19104, USA. \\ e-mail:mgrossma@mail.med. \\ upenn.edu
}

Accurate ante mortem diagnosis in frontotemporal lobar degeneration (FTLD) is crucial to the development and implementation of etiology-based therapies. Several neurodegenerative disease-associated proteins, including the major protein constituents of inclusions in Alzheimer's disease (AD) associated with amyloid-beta $\left(A \beta_{1-42}\right)$ plaque and tau neurofibrillary tangle pathology, can be measured in cerebrospinal fluid (CSF) for diagnostic applications. Comparative studies using autopsy-confirmed samples suggest that CSF total-tau (t-tau) and $A \beta_{1-42}$ levels can accurately distinguish FTLD from $A D$, with a high t-tau to $A \beta_{1-42}$ ratio diagnostic of $A D$; however, there is also an urgent need for FTLD-specific biomarkers. These analytes will require validation in large autopsy-confirmed cohorts and face challenges of standardization of within- and between-laboratory sources of error. In addition, CSF biomarkers with prognostic utility and longitudinal study of CSF biomarker levels over the course of disease are also needed. Current goals in the field include identification of analytes that are easily and reliably measured and can be used alone or in a multi-modal approach to provide an accurate prediction of underlying neuropathology for use in clinical trials of disease modifying treatments in FTLD. To achieve these goals it will be of the utmost importance to view neurodegenerative disease, including FTLD, as a clinicopathological entity, rather than exclusively a clinical syndrome.

Keywords: cerebrospinal fluid, biomarker, tau, $A \beta_{1-42}$, frontotemporal dementia, primary progressive aphasia, Alzheimer's disease

\section{INTRODUCTION}

Most neurodegenerative diseases are characterized by specific abnormally-modified protein aggregates, with resulting neuronal cell loss and gliosis. The gold standard for diagnosis is microscopic examination at autopsy; however, there is considerable variability of clinical manifestations associated with underlying

\footnotetext{
Abbreviations: FTLD, frontotemporal lobar degeneration; AD, Alzheimer's disease; $\mathrm{A} \beta$, amyloid-beta; CSF, cerebrospinal fluid; CNS, central nervous system; FTLD-tau, FTLD with tau pathology; TDP-43, TAR DNA binding protein-43; FTLD-TDP, FTLD with TDP pathology; PiD, Pick's disease; CBD, corticobasal degeneration; PSP, progressive supranuclear palsy; pathogenic MAPT mutationsFTDP-17, FTD and parkinsonism linked to chromosome 17; ALS, amyotrophic lateral sclerosis; FUS, fused-in-sarcoma protein; FTLD-FUS, FTLD with FUS pathology; FTLD-UPS, FTLD with tau- and TDP-43-negative ubiquitinated inclusions; FTLD-ni, FTLD in the absence of significant neuropathological inclusions; GRN, progranulin gene; MAPT, tau gene; C9orf72, C9orf72 gene; $V C P$, valosin-containing protein gene; TARDBP, TDP-43 gene; FTLD-ALS, clinical FTLD with ALS; CHMP2B, charged mutlivesciular body protein 2B gene; bvFTD, behavioral-variant frontotemporal dementia; PPA, primary progressive aphasia; lvPPA, logopenic-variant PPA; svPPA, semantic-variant PPA; naPPA, non-fluent aggramatic variant PPA; CBS, corticobasal syndrome; $A \beta_{1-42}, \beta$-amyloid; MCI, mild cognitive impairment; $\mathrm{t}$-tau, total-tau; $\mathrm{p}$-tau, phosphorylated-tau; $\mathrm{p}$-tau ${ }_{181}$, phosphorylated tau at serine 181 ; p-tau 231 , phosphorylated tau at threonine 231; ELISA, enzyme-linked immunosorbent assay; xMAP, luminex flow immunoassay; MRI, magnetic resonance imaging; DIAN, dominantly-inherited AD network; MTBD, microtubule-binding domain; DTI, diffusion-tensor imaging; GM, gray matter; GWAS, genome-wide association studies.
}

neuropathological diagnoses, as clinical symptoms most often reflect the regional burden of pathology within the central nervous system (CNS) rather than the specific underlying proteinopathy. This is especially true in the heterogeneous family of frontotemporal lobar degeneration (FTLD) clinical syndromes.

Two main pathologic FTLD subtypes exist (Figures 1A, 2): cases with inclusions formed from the microtubule-binding protein tau (FTLD-tau) and those with TAR DNA binding protein43 (TDP-43) pathology (FTLD-TDP) (Mackenzie et al., 2010). FTLD-tau includes the following tauopathies (Figures 2A-D): Pick's disease (PiD), corticobasal degeneration (CBD), progressive supranuclear palsy (PSP), FTD and parkinsonism linked to chromosome 17 (pathogenic MAPT mutations; FTDP-17), and unclassifiable tauopathies (Mackenzie et al., 2010). FTLDTDP (Figures 2E-G) can be subdivided into four subtypes (A-D) based on the morphology and distribution of lesions (Mackenzie et al., 2011) and can also be associated with TDP-43 inclusions in the anterior horn of the spinal cord and gliosis of the corticospinal tracts, suggesting a continuum of FTLD with amyotrophic lateral sclerosis (ALS; FTLD-ALS) (Geser et al., 2008, 2009). A smaller number of FTLD cases are associated with inclusions of another DNA-binding protein, fused-in-sarcoma protein (FUS; FTLD-FUS), or other rare, less-defined pathologies (FTLD-UPS, FTLD-ni) (Mackenzie et al., 2010). The major 


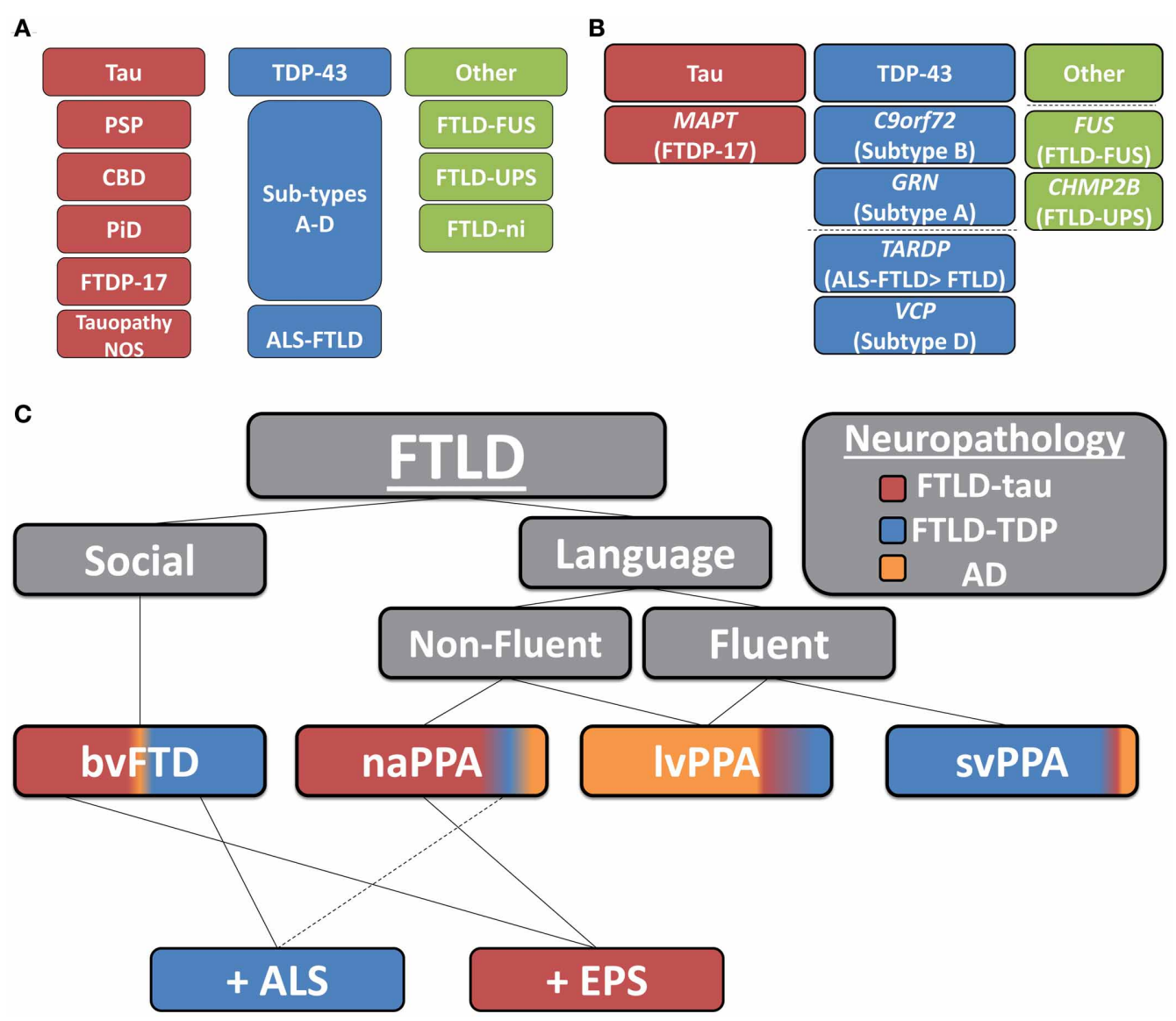

FIGURE 1 | Clinicopathological and genetic associations in FTLD.

(A) Neuropathological classification of FTLD-tau and FTLD-TDP subtypes (PSP, progressive supranuclear palsy; CBD, corticobasal degeneration; PiD, Pick's disease; FTDP17, frontotemporal dementia with Parkinsonism linked to chromosome 17; Tauopathy NOS, unclassifiable tauopathy; Subtypes A-D, morphological subtypes of FTLD-TDP; ALS-FTLD, amyotrophic lateral sclerosis with FTLD-TDP; FTLD-FUS, FTLD with fused in sarcoma protein inclusions; FTLD-UPS, FTLD with tau- and TDP-43-negative ubiquitinated inclusions; FTLD-ni, FTLD in the absence of significant neuropathological inclusions), (B) pathogenic mutation associations with underlying neuropathology (dashed-line separates less common molecular etiologies of FTLD; MAPT, tau resulting in FTDP-17; C90rf72, pathogenic hexanucleotide expansion resulting in FTLD and/or ALS associated with FTLD-TDP B; GRN, progranulin resulting in FTLD-TDP type A; TARDP, TDP-43 resulting in ALS \pm FTLD and less commonly FTLD; VCP, valosin-containing protein resulting in inclusion body myopathy with Paget's disease of bone and frontotemporal dementia with FTLD-TDP subtype D; FUS, fused-in sarcoma protein resulting in FTLD-FUS; and CHMP2B, charged mutlivesciular body protein 2B resulting in FTLD-UPS), (C) clinicopathological correlations of FTLD (colored regions of clinical syndromes represent relative percentages of neuropathological subtypes found in autopsy studies; AD, Alzheimer's disease; bvFTD, behavioral variant of FTLD; PPA, primary progressive aphasia; SVPPA, semantic variant PPA; naPPA, nonfluent agrammatic variant PPA; IVPPA, logopenic variant PPA; +ALS, co-morbid amyotrophic lateral sclerosis; + EPS, co-morbid extra-pyramidal Parkinsonian symptoms: i.e., features of akinetic-rigid syndromes of PSP or corticobasal syndrome). genetic etiologies resulting in FTLD are exclusively associated with specific underlying neuropathologies (Figure 1B), despite heterogeneous expression of FTLD clinical syndromes, and include pathogenic mutations in the gene for progranulin (GRN) (Baker et al., 2006; Cruts et al., 2006), tau (MAPT) (Hutton et al., 1998), and C9orf72 (C9orf72) (Dejesus-Hernandez et al., 2011; Renton et al., 2011). Less common genetic etiologies of FTLD include: valosin-containing protein $(V C P)$ resulting in inclusion body myopathy with Paget's disease of bone and frontotemporal dementia with FTLD-TDP subtype D neuropathology, TARDBP coding for TDP-43 protein and causing ALS or ALS-FTLD (rarely FTLD-TDP alone), $C H M P 2 B$ coding for charged mutlivesciular body protein $2 \mathrm{~B}$ and resulting in FTLD-UPS, and mutations in FUS causing FTLD-FUS (Figure 1B) (Mackenzie et al., 2010).
Clinically, FTLD can be broadly divided into two main subtypes, those with predominant behavioral and social comportment disorder (behavioral-variant frontotemporal dementia, bvFTD) (Rascovsky et al., 2011) and those with primary language disturbances (primary progressive aphasia, PPA) (Mesulam, 1982, 2001). Among PPA patients, three subgroups have been recently divided (Gorno-Tempini et al., 2011) into the logopenic (lvPPA) (Gorno-Tempini et al., 2004, 2008), semantic (svPPA) (Hodges and Patterson, 2007), and non-fluent aggramatic variant (naPPA) (Turner et al., 1996). Clinicopathological correlations of these syndromes are complex (Josephs, 2008; Grossman, 2010). For example, large studies of autopsy-confirmed FTLD (behavioral and aphasic variants) find roughly equal numbers of FTLD-tau and FTLD-TDP (Hodges et al., 2004; Kertesz et al., 


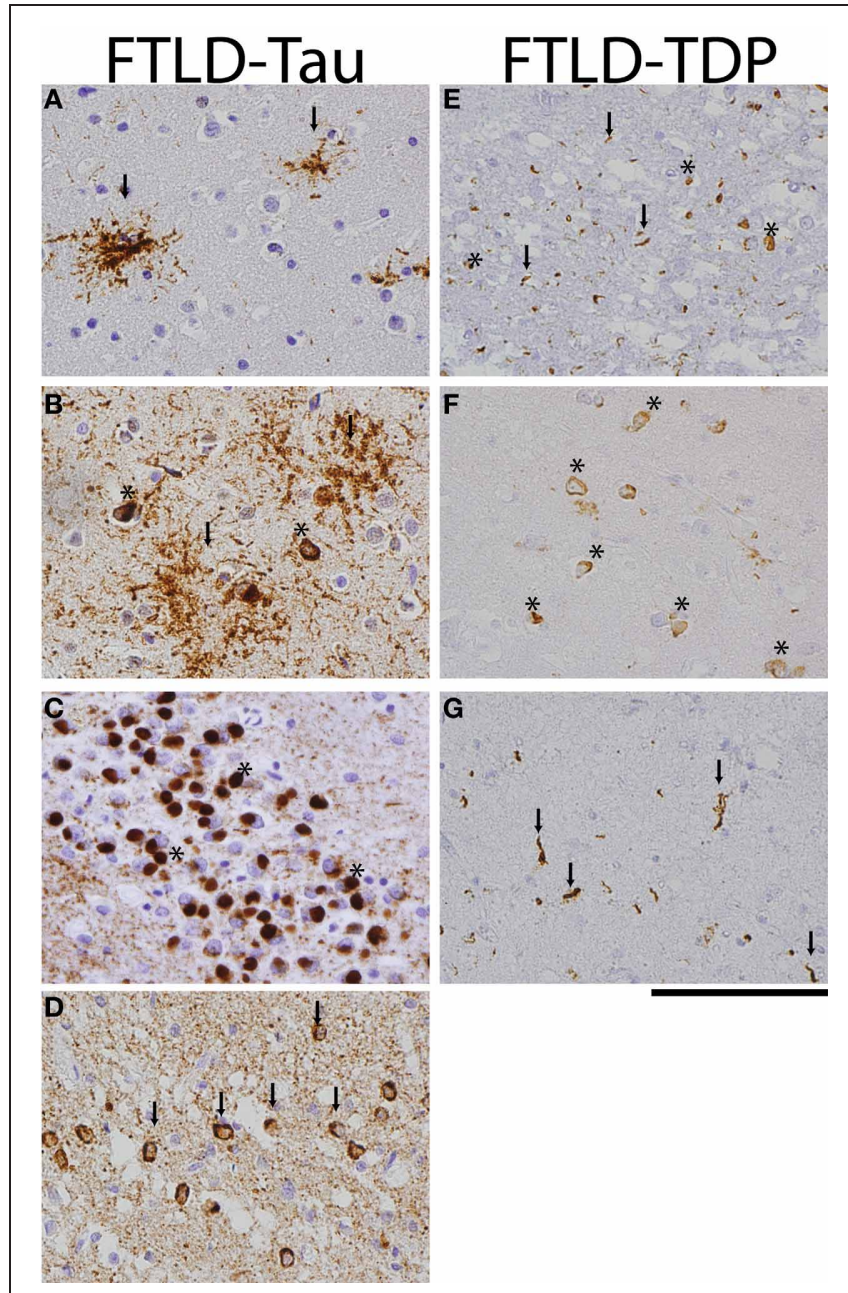

FIGURE 2 | FTLD-Tau and FTLD-TDP histology. Photomicrographs of FTLD-tau (A-D) and FTLD-TDP (E-G) visualized with immunohistochemistry (PHF-1 and pTDP 409/410 for tau and TDP, respectively). (A) PSP frontal cortex with tau-positive tufted astrocytes (arrows), (B) CBD temporal cortex with diffuse astrocytic plaques (arrows) and neuronal tangles (asterisks), (C) Pick's disease with round tau-positive Pick bodies (asterisks) in the dentate nucleus of the hippocampus, (D) FTDP-17 case with p.P301L pathogenic mutation with tau-positive neuronal tangles (arrows) and diffuse neuropil threads in temporal cortex, (E) FTLD-TDP subtype A with cytoplasmic neuronal inclusions (asterisks) and short dystrophic neurites (arrows) in superficial layers of frontal cortex, (F) FTLD-TDP subtype B with prominent cytoplasmic inclusions (asterisks) in deep temporal cortical layer, and (G) long dystrophic neurites (arrows) in superficial layers of mid-frontal cortex of a patient with FTLD-TDP subtype C. Scale bar $=100 \mu \mathrm{m}$.

2005; Knopman et al., 2005; Shi et al., 2005; Forman et al., 2006). Furthermore, a primary neuropathological diagnosis of Alzheimer's disease (AD) has been found in up to 30\% of autopsyconfirmed clinically defined FTLD cohorts (Kertesz et al., 2005; Knopman et al., 2005; Forman et al., 2006; Knibb et al., 2006). Examination of focal presentations of $\mathrm{AD}$ found it to be the primary diagnosis in $7 \%$ of bvFTD, $44 \%$ of naPPA, $10 \%$ of svPPA, and $50 \%$ of the extrapyramidal and cognitive disorder, corticobasal syndrome (CBS) patients (Alladi et al., 2007). Others have also found a substantial proportion of AD in PPA cases (Forman et al., 2006; Knibb et al., 2006) especially in lvPPA (Grossman et al., 2008; Mesulam et al., 2008; Grossman, 2010) and also CBS (Lee et al., 2011). Thus, differentiation of AD and FTLD spectrum disorders poses a serious diagnostic challenge for clinicians.

Within the FTLD neuropathological spectrum, examination of the specific clinical subtypes finds varying degrees of association with FTLD-tau and FTLD-TDP (Figure 1C). FTLD-tau has been overrepresented in some naPPA cohorts (Hodges et al., 2004; Josephs et al., 2006a,b; Knibb et al., 2006; Snowden et al., 2007; Mesulam et al., 2008; Grossman et al., 2012), especially when associated with apraxia of speech (Josephs et al., 2006a; Snowden et al., 2007) and svPPA has been predominantly associated with TDP-43 pathology (Hodges et al., 2004; Josephs et al., 2006a; Snowden et al., 2007; Grossman et al., 2008); while bvFTD contains similar proportions of FTLD-tau and FTLD-TDP (Forman et al., 2006; Josephs et al., 2006b; Snowden et al., 2007). Extrapyramidal symptoms may predict a tauopathy (Forman et al., 2006; Josephs et al., 2006b) while co-morbid ALS is almost certainly due to TDP-43 aggregation (Shi et al., 2005; Forman et al., 2006; Josephs et al., 2006b). Clinicopathological associations from these large autopsy studies are summarized in Figure 1C.

A major challenge in the development and implementation of disease-modifying therapy in FTLD is the accurate identification of the neuropathological diagnosis during life, including differentiation from $\mathrm{AD}$, so that patients may be triaged to the appropriate protein-targeted therapy (i.e., tau or TDP-43 targeted agents).

Biofluid biomarkers have the potential to optimize diagnostic accuracy and detect disease earlier in the course of an illness and possibly pre-symptomatically, such as prior to structural changes of neurodegeneration seen on neuroimaging ( $\mathrm{Hu}$ et al., 2010a; Jack et al., 2010), making further exploration in this area promising for the development of disease modifying treatments. In addition, some clinical measures of disease progression in FTLD, including functional scales, may be limited by floor- and ceiling-effects (Knopman et al., 2008), so biofluid biomarkers are potentially attractive surrogate end points for use in clinical trials (Boxer et al., 2012b). The cerebrospinal fluid (CSF) is relatively easy to obtain and contains a direct connection to the pathological milieu in central nervous system, making it a desirable biofluid for study. In this review we will discuss the current state of CSF biomarker research in FTLD in terms of differentiation from AD and future directions and challenges for the field in development of FTLD-specific biomarkers.

\section{ALZHEIMER'S DISEASE RELATED CSF BIOMARKERS: A $\beta_{1-42}$ AND tau STUDIES IN ALZHEIMER'S DISEASE}

As a first step in biofluid-based biomarker assessment of neurodegenerative disease, it is valuable to distinguish broadly between $\mathrm{AD}$ and FTLD. CSF values of the major constituents of AD pathology, tau and $\beta$-amyloid, $\left(A \beta_{1-42}\right)$ have been widely studied using immune-based analytical platforms in $\mathrm{AD}$ and amnestic mild cognitive impairment (MCI) patients, with lower $A \beta_{1-42}$ values and higher levels of total- and phosphorylated-tau (t-tau, p-tau) compared with controls across multiple large studies (Shaw et al., 
2009, 2011; De Meyer et al., 2010; Trojanowski et al., 2010; Weiner et al., 2010). Furthermore, our group has shown prognostic utility of these markers by accurately predicting MCI conversion to AD (Shaw et al., 2009; De Meyer et al., 2010).

The majority of atypical clinical presentations of AD in earlyonset patients consisting of predominantly visuo-spatial difficulties (i.e., consistent with poster cortical atrophy) or asymmetric apraxia/rigidity (i.e., consistent with CBS) may have a similar CSF biomarker profile to that of typical amnestic-AD (De Souza et al., 2011; Seguin et al., 2011), with a further elevated t-tau level in one study (Koric et al., 2010). Elevated CSF t-tau and low A $\beta_{1-42}$ levels have also been described in some PPA patients (i.e., lvPPA) (Bibl et al., 2011; De Souza et al., 2011) most likely due to underlying $\mathrm{AD}$ neuropathology in these individuals; however, to our knowledge no autopsy-confirmed studies of atypical clinical AD presentations have been performed.

The exact relationship between $\mathrm{AD}$ neuropathologic change (i.e., tau neurofibrillary pathology and $A \beta_{1-42}$ extracellular plaques) and observed measurement of these analytes in CSF is unclear; however, the total tau level is thought to reflect underlying neurodegeneration and neuron loss, as elevations are also seen in other CNS insults (Otto et al., 1997; Hesse et al., 2000; Jin et al., 2006; Ost et al., 2006; Krut et al., 2013). Lower A $\beta_{1-42}$ CSF levels may be the result of sequestration of soluble interstitial brain $\mathrm{A} \beta_{1-42}$ into extracellular plaques as there is an inverse correlation of CSF $A \beta_{1-42}$ levels and the degree of cortical plaque pathology (Tapiola et al., 2009; Patel et al., 2012; Seppala et al., 2012) and in vivo neuroimaging evidence of amyloidosis (Fagan et al., 2006). Phosphorylated epitopes of tau (p-tau) can be measured in CSF as well; while most phospho-epitopes of tau are also found in healthy non-diseased brains and are not AD-specific, pathological tau species overall are highly phosphorylated in AD (Matsuo et al., 1994) and this altered state reflects the elevated levels of p-tau seen in AD. The most commonly studied p-tau epitopes are serine 181 ( $\mathrm{p}$-tau 181 ) (Vanmechelen et al., 2000), and threonine $231\left(\mathrm{p}-\mathrm{tau}_{231}\right)$ (Buerger et al., 2002a,b).

\section{STUDIES IN FRONTOTEMPORAL LOBAR DEGENERATION}

FTLD is not characterized pathologically by cerebral $A \beta_{1-42}$ amyloidosis, and only FTLD-tau is characterized by significant tau inclusions. From this perspective, measures of CSF t-tau and $\mathrm{A} \beta_{1-42}$ may have helpful diagnostic utility in excluding $\mathrm{AD}$ neuropathology. Indeed, in clinically-defined cohorts $\mathrm{AD}$ cases have higher levels of t-tau, $\mathrm{p}$-tau $\tan _{18}$ and lower levels of $\mathrm{A} \beta_{1-42}$ compared to FTLD and controls in group-wise comparisons (Blennow et al., 1995; Arai et al., 1997; Green et al., 1999; Sjogren et al., 2000a, 2001; Vanmechelen et al., 2000; Riemenschneider et al., 2002; Clark et al., 2003; Pijnenburg et al., 2004, 2007; Schoonenboom et al., 2004, 2012; Engelborghs et al., 2006; Bibl et al., 2007, 2011; Kapaki et al., 2008; Verwey et al., 2010; De Souza et al., 2011; Gabelle et al., 2011; Van Harten et al., 2011).

A major challenge in FTLD CSF biomarker studies is the heterogeneity of the condition (Figure 1), making autopsyconfirmation of diagnostic classification a crucial issue. As mentioned previously, up to $30 \%$ of clinically-defined FTLD cohorts may have underlying $\mathrm{AD}$ neuropathologic change as the etiology of their symptoms (Kertesz et al., 2005; Knopman et al.,
2005; Forman et al., 2006; Knibb et al., 2006) and contamination with these atypical $\mathrm{AD}$ cases could influence results significantly. Indeed, examination of diagnostic accuracy of CSF t-tau and $\mathrm{A} \beta_{1-42}$ in a large autopsy-confirmed dementia cohort found that use of the clinical diagnosis, rather than neuropathological diagnosis as the gold standard for biomarker performance resulted in a 10-20\% underestimation of biomarker accuracy (Toledo et al., 2012). Furthermore, since 1995 there has been over a 10 -fold increase in the number of FTLD manuscripts published (NLM/NIH, 2012) and due to this exponential increase in research in the field and our expanding knowledge of FTLD, clinical criteria (Gorno-Tempini et al., 2011; Rascovsky et al., 2011) have evolved resulting in refinement of our clinical definitions. Indeed, the emergence of the new clinical variant of PPA, lvPPA (Gorno-Tempini et al., 2008, 2011), which is most often associated with AD neuropathology (Mesulam et al., 2008; Rabinovici et al., 2008; Grossman, 2010) (Figure 1C), and therefore suggested to be excluded from FTLD clinical trials (Knopman et al., 2008), could influence group-wise CSF tau and $\mathrm{A} \beta_{1-42}$ results. Thus, the makeup of clinical cohorts used in earlier studies may not be entirely translatable to newer studies, limiting the meaningful interpretation of the literature of clinically-derived cohorts.

As such, study of autopsy/genetic-confirmed cases has been a focus for our center. In an early study of autopsy-confirmed cases by our group, $\mathrm{AD}$ was differentiated from a mixed dementia cohort (including 13 FTLD cases) with reasonable sensitivity (72\%) and specificity (69\%) using CSF t-tau levels (Clark et al., 2003). Focused analysis of FTLD (with autopsy confirmation in 9 cases) in a later study found lower levels of t-tau and higher levels of $A \beta_{1-42}$ than $A D$, and roughly $30 \%$ of FTLD cases had significantly decreased t-tau from controls (Grossman et al., 2005). In a follow-up large autopsy/genetically confirmed FTLD series $(n=30)$ t-tau levels were significantly lower in FTLD than AD, while similar to controls on group-wise comparison; individualcase analysis revealed that a considerable subset of FTLD patients had markedly low t-tau values (Bian et al., 2008). Interestingly, FTLD cases with substantially lower t-tau levels included both FTLD-tau and FTLD-TDP (Bian et al., 2008), although a nonsignificant trend was found for lower t-tau in FTLD-tau (Hu et al., 2011). Furthermore, FTLD was differentiated from AD with high accuracy using the $\mathrm{t}-\mathrm{tau} / \mathrm{A} \beta_{1-42}$ ratio; that is, FTLD cases had a lower ratio (lower t-tau and higher $\mathrm{A} \beta_{1-42}$ ) (Bian et al., 2008).

Measurement of these analytes in the CSF in most studies utilizes one of two immune-based platforms: enzymelinked immunosorbent assay (ELISA; Innotest, Innogenetics), and a multiplex assay based on flow-cytometry of antibodycoated fluorescent beads (INNO-BIA AlzBio3 xMAP; Luminex, Innogenetics). Absolute values obtained from these platforms differ because the coefficient of variance $(\% \mathrm{CV})$ with the xMAP Luminex platform is much narrower than with ELISA, but they are highly correlated (Olsson et al., 2005; Lewczuk et al., 2009; Fagan et al., 2011; Wang et al., 2012) and have similar levels of diagnostic accuracy for AD (Fagan et al., 2011; Wang et al., 2012) and differentiating AD from FTLD (Toledo et al., 2012). Thus, values from one platform can be effectively transformed into equivalent units of the other using a conversion 
factor (Fagan et al., 2011; Wang et al., 2012). Indeed, we were able to transform values obtained from ELISA to equivalent xMAP units using linear regression to create a larger autopsy/geneticconfirmed FTLD dataset and help confirm our pervious observations of the diagnostic utility of the t-tau/A $\beta_{1-42}$ ratio to differentiate FTLD from AD (Irwin et al., 2012b). Maximizing available data is crucial for these extremely valuable and well-annotated research samples. In summary, in multiple large-scale autopsyconfirmed studies we have demonstrated the diagnostic utility of CSF t-tau, p-tau, and $A \beta_{1-42}$ in differentiation of AD and FTLD (Bian et al., 2008; Irwin et al., 2012b; Toledo et al., 2012).

Few other CSF studies have used autopsy-confirmed cohorts of FTLD patients (Table 1). One study included 10 autopsyconfirmed FTLD patients and found similar results of lower t-tau and p-tau 181 levels in FTLD compared with AD, with high diagnostic accuracy of p-tau 181 (Koopman et al., 2009). Another study including 12 confirmed FTLD patients described "slightly elevated tau levels" in several patients compared to an agedependent reference range and low compared to the majority of AD cases (Brunnstrom et al., 2010). Neuropathological subgroups of FTLD (FTLD-TDP, $n=5$ and FTLD-tau, $n=7$ ) had similar mean values, with $4 / 12$ patients below the reference limit by $>70 \mathrm{pg} / \mathrm{ml}$ (Brunnstrom et al., 2010). Thus, this study also found a subset of individual FTLD patients with lower than normal t-tau levels. The diagnostic utility of t-tau/A $\beta_{1-42}$ in differentiating FTLD was not systematically explored in this small group of $\mathrm{AD}$ cases $(n=8)$. Finally, to our knowledge the only additional studies utilizing autopsy-confirmed FTLD cohorts included a small number of FTLD cases $(<10)$ in a non-AD category, with no direct comparison of FTLD and AD (Engelborghs et al., 2008; Tapiola et al., 2009; Schoonenboom et al., 2012). Thus, further study is required in large prospective, autopsy-confirmed samples to confirm our observations.

The higher $\mathrm{A} \beta_{1-42}$ in FTLD compared to $\mathrm{AD}$ most likely reflects the absence of significant cerebral amyloidosis while the biological basis for observed low CSF t-tau in some FTLD patients is uncertain. One possibility is related to cortical tau depletion (Zhukareva et al., 2001, 2003; Grossman et al., 2005) through sequestration into the neuronal and glial inclusions in the absence of significant extracellular tau pathology (FTLD-tau) Dickson, 2004, such as extracellular "ghost tangles" as seen in AD (Schmidt et al., 1988), or altered post-translational stability of tau in FTLDTDP (Zhukareva et al., 2001, 2003); furthermore, CSF t-tau does appear related to underlying FTLD pathophysiology as t-tau levels

Table 1 | Comparative studies of CSF biomarkers in autopsy/genetic-confirmed FTLD and AD cohorts.

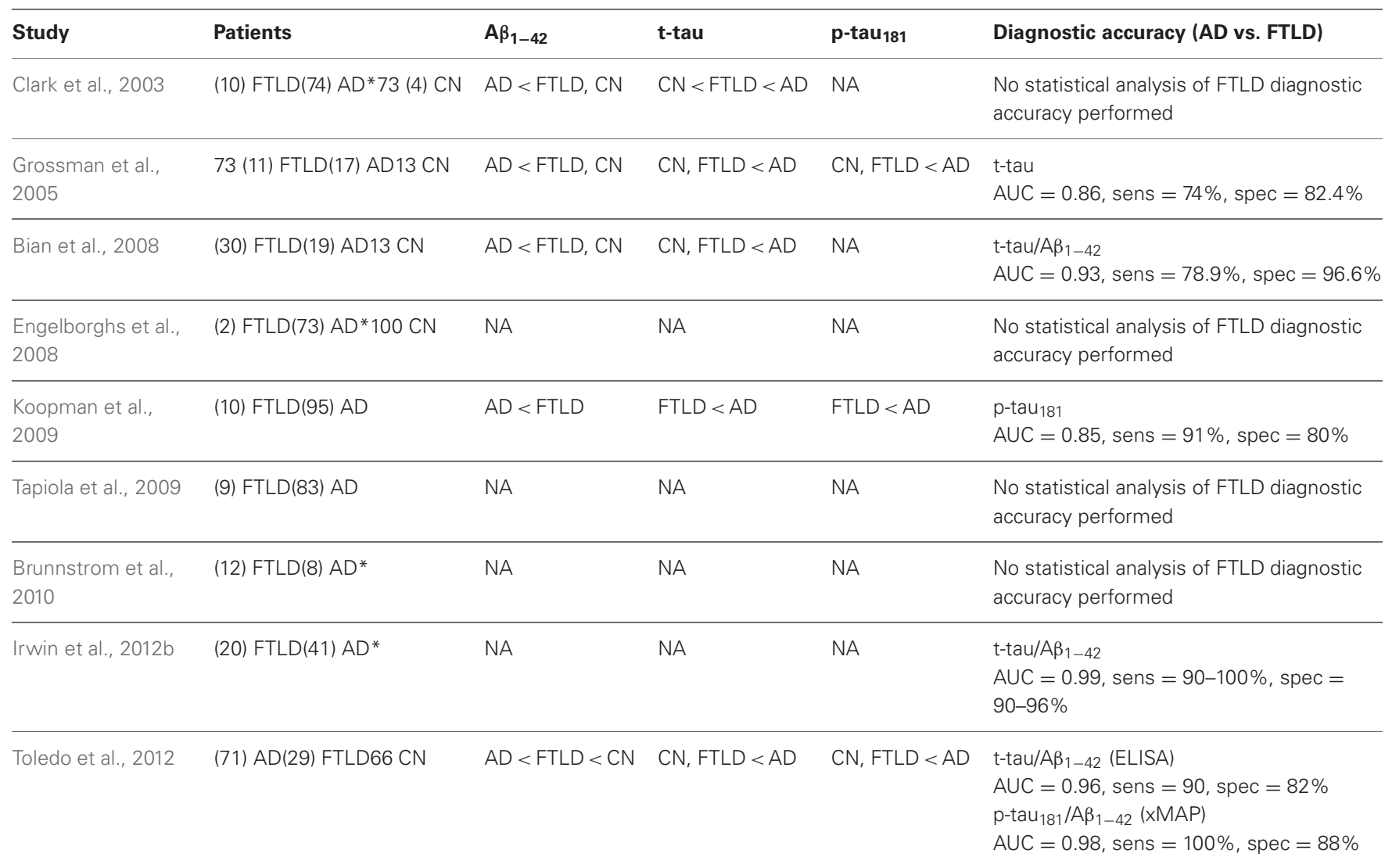

Other diagnostic groups that may be present in some studies are omitted and only direct comparisons of FTLD group to AD or CN are reported. " $<$ " or " $>$ " denotes significant difference between groups and "," denotes non-significant difference between groups, () denotes autopsy/genetic confirmed cohort. $C N$, non-demented controls; * , AD group contains cases with co-morbid Lewy Body or Vascular Disease; NA, Not assessed; AUC, Area under the curve for receiver operating curve analysis; ELISA, enzyme-linked immunosorbent assay; XMAP, luminex multiplex assay. 
in FTLD patients correlated to areas of frontal and temporal cortical atrophy on magnetic resonance imaging (MRI) (Grossman et al., 2005; McMillan et al., 2013). Further study of CSF protein dynamics in animal models of disease may help clarify these seemingly discordant associations of low tau levels with underlying neuropathology in FTLD-tau and FTLD-TDP.

Despite the clear distinction of t-tau and $A \beta_{1-42}$ levels between $\mathrm{AD}$ and FTLD, there is more variability in the literature for the relationship of these markers in FTLD compared with non-demented controls (Table 1). There are several reasons for these discrepancies; first, even in most autopsy-based studies, autopsy data on controls is lacking (Table 1) and a significant proportion of non-demented elderly can have underlying $\mathrm{AD}$ neuropathology (Davis et al., 1999), and thus influence CSF analyte measures. Next, even with pathologic confirmation, patient classification in FTLD is challenging, as another potential confounding issue is the presence of mixed pathologies in dementia patients. Indeed, our group has shown in a large autopsyconfirmed sample that mixed pathology is present in roughly $30 \%$ of cases, and that FTLD patients with significant AD neuropathologic change can influence the CSF t-tau and $A \beta_{1-42}$ levels, causing higher t-tau and lower $A \beta_{1-42}$ in cases with mixed FTLD and AD pathology compared to "pure" FTLD (Toledo et al., 2012). Additionally, a recent largely clinically-defined cohort study found an AD CSF biomarker profile in 30\% of FTLD (Schoonenboom et al., 2012) which may be due, in part, to mixed pathology or inclusion of atypical AD cases mimicking the FTLD clinical syndrome (Toledo et al., 2012). Thus, the use of autopsy-confirmed samples is essential for in-depth study and validation of the diagnostic accuracy of potential biomarkers in FTLD.

Finally, variability in measurement between studies is another potential issue as significant variation between centers in absolute values measured in "spiked" pooled CSF control samples with known concentrations of analyte has been described (Shaw et al., 2011). These discrepancies are most likely due to sources of variation in CSF collection, handling and storage (pre-analytical), equipment, reagents and methods of analysis (analytical), and data management and interpretation (postanalytical) (Mattsson et al., 2011). For these reasons, large scale studies of measurement precision of these analytes and coordinated multi-center quality control programs with standard operating procedures to minimize these sources of variation have been conducted (Mattsson et al., 2011; Shaw et al., 2011).

Despite these issues, we have demonstrated (Bian et al., 2008; Irwin et al., 2012b; Toledo et al., 2012) that these AD-specific analytes ( $\mathrm{t}$-tau to $\mathrm{A} \beta_{1-42}$ ratio) may perform within the range of sensitivity and specificity $(>80 \%)$ for use in clinical trials (Trojanowski and Growdon, 1998) to differentiate FTLD from $\mathrm{AD}$; however, these analytes are not as effective for differentiation of FTLD from normal controls (Bian and Grossman, 2007; Toledo et al., 2012). Although patients may present with decompensated psychiatric issues or other non-progressive non-degenerative etiologies resembling FTLD (phenocopy syndrome) (Kipps et al., 2010), these patients may be identified with serial clinical exams and neuroimaging (Kipps et al., 2010). The more urgent need is for FTLD-specific biomarkers and those that can differentiate between the two major neuropathologic subtypes (FTLD-tau and FTLD-TDP) (Hu et al., 2011).

\section{FUTURE DIRECTIONS FURTHER STUDY OF CSF tau AND A $\boldsymbol{\beta}_{\mathbf{1}-42}$}

Previous work in large cross-sectional studies in $\mathrm{AD}$ suggests a temporal progression of dynamic biomarker change in $\mathrm{AD}$ (Jack et al., 2010, 2012), as $\mathrm{A} \beta_{1-42}$ amyloidosis, and resultant lower CSF $A \beta_{1-42}$, is thought to occur decades before clinical symptoms emerge in $\mathrm{AD}$, while increased CSF t-tau is thought to be a later event in disease progression and correlates more closely with cognitive decline. It is likely that t-tau, p-tau and potential novel CSF biomarkers could display similar changes throughout the course of disease in FTLD and could correlate with clinical symptoms. Few studies have examined the change in CSF biomarkers over time or their relation to clinical symptoms. One study included a follow up CSF analysis in one FTLDtau patient, with similar t-tau and $A \beta_{1-42}$, roughly 18 months between CSF collections (Brunnstrom et al., 2010). Interestingly, a recent study of bvFTD patients found a significant correlation with $A \beta_{1-42}$ levels and cognitive performance, even after removal of patients with CSF profile suggestive of $\mathrm{AD}$ neuropathology (Koedam et al., 2012). These results could suggest an influence of co-morbid AD neuropathology; however autopsy information in these cases was lacking. Other studies in clinical series without autopsy confirmation found no association of these markers and clinical measures or disease severity (Riemenschneider et al., 2002; Engelborghs et al., 2006; De Souza et al., 2011). Further study of clinical correlates of CSF biomarkers and longitudinal profiles of CSF analyte change throughout the course of disease will be helpful.

Similar to the dominantly-inherited AD network (DIAN) initiative to study patients with known pathogenic mutations to cause AD (Bateman et al., 2012), study of prodromal FTLD patients with pathogenic mutations may provide additional insights into the temporal sequence of biomarkers in FTLD (Boxer et al., 2012a). Furthermore, CSF analyte levels in symptomatic patients with genetic forms of FTLD have not been explored in detail and could potentially differ from sporadic cases. Indeed, we found a more rapid rate of progression in cognitive measures corresponding to more severe neurodegeneration in C9orf72-associated FTLD (Irwin et al., 2013) and others have described unique neuroimaging patterns of atrophy across different genetic forms of FTLD (Whitwell et al., 2012). This evidence of biologic differences in genetic and sporadic FTLD suggest alterations in CSF biomarker profiles are also a possibility, although one study found similar levels of CSF tau and $A \beta_{1-42}$ in genetically-confirmed FTDP-17 $(n=9)$ compared to sporadic FTLD $(n=17)$ (Rosso et al., 2003).

\section{DEVELOPMENT OF FTLD-SPECIFIC BIOMARKERS}

In the context of disease-modifying therapies targeting a specific histopathologic abnormality, an important goal is to distinguish between FTLD due to TDP-43 and FTLD due to tau. Exploratory analyses for novel biomarkers that have diagnostic utility in FTLD are ongoing and include several basic approaches. 
First, measurement of biologically relevant molecules is the most straightforward approach, as tau and $\mathrm{A} \beta_{1-42}$ have been successful biomarker candidates in $\mathrm{AD}$. Using this rationale, the two most obvious candidates for FTLD-specific biomarkers are TDP43 progranulin. Indeed, TDP-43 has been detected in human CSF (Steinacker et al., 2008; Kasai et al., 2009) and serum (Foulds et al., 2008), suggesting elevated levels may occur in some patients with TDP-43 proteinopathies, but initial studies show limited diagnostic accuracy. Low serum progranulin may identify FTLD patients with a pathogenic GRN mutation resulting in progranulin haploinsufficiency (Ghidoni et al., 2008), which could be useful in monitoring potential progranulinreplacing therapies in development for FTLD (Boxer et al., 2012b).

Other biologically relevant potential biomarkers for FTLD include specific isoforms or neoepitopes of tau. Tau undergoes multiple post-translational modifications thought to contribute to tangle formation. Indeed, we found acetylation of tau at a specific residue in the microtubule-binding domain (MTBD) to be exclusively found in tauopathies, providing promise for this epitope as a useful marker of AD and FTLD-tau (Cohen et al., 2011; Irwin et al., 2012a). Translating these immunohistochemical observations to clinical assays may prove difficult, as levels of tau in CSF are near the lower limits of biologic detection (Hampel et al., 2010) limiting the further identification of a specific subset of tau in the form of a neoepitope; although one group has found promising evidence for diagnostic utility of specific C-truncated isoforms of tau in PSP through immunoprecipitation and western blotting techniques (Borroni et al., 2008, 2009) and others have developed assays to measure 3- and 4R tau in CSF (Luk et al., 2012). Alternativelytruncated forms of $\mathrm{A} \beta_{1-42}$ may also have diagnostic importance in FTLD (Pijnenburg et al., 2007; Bibl et al., 2011, 2012; Gabelle et al., 2011) and cytoskeletal proteins, such as neurofilament have also been explored (Sjogren et al., 2000b; De Jong et al., 2007). These potential biomarkers warrant further study and validation.

Another, possible approach is to screen a large number of potential analytes without an a priori biologic rationale in a proteomic analysis of CSF in FTLD. Indeed, using an immune-based multiplex approach our group found promising CSF biomarker candidates to differentiate FTLD-TDP and FTLD-tau with high sensitivity and specificity, but these candidate analytes need further study to confirm their utility as FTLD biomarkers (Hu et al., 2010b). Finally, other non-immune based methods, such as massspectrometry are also being explored to identify novel biofluid biomarkers in FTLD (Mattsson et al., 2008).

Potential FTLD-specific biofluid biomarkers will be faced with the same challenges of testing reliability and sources of variation (i.e., analytical, pre-/post-analytical) currently experienced by CSF t-tau and $A \beta_{1-42}$ measurements. As such, coordinated and cooperative efforts between multiple centers will undoubtedly be necessary to help validate potential FTLD-specific CSF biomarkers prior to clinical use.

Most likely, a multimodal assessment incorporating potential novel biofluid biomarker values with clinical, neuroimaging and genetic markers may be the most effective approach to accurately identify FTLD subtypes. Neuropsychological testing can help differentiate AD from FTLD (Rascovsky et al., 2008; Libon et al., 2011) as routine cognitive measures may not be sensitive enough to detect the behavioral and language deficits in FTLD. Indeed, our group has explored quantitative approaches to language (Ash et al., 2006, 2009; Gunawardena et al., 2010) and social cognition (Massimo et al., 2009, 2013; Grossman et al., 2010; Eslinger et al., 2012; McMillan et al., 2012b) to examine brain-behavior relationships and improve diagnostic accuracy in FTLD. Neuroimaging is another potential method with diagnostic utility alone, or as an adjunct to clinical and biofluid biomarkers in FTLD; we have found combining neuropsychological testing and MRI can improve diagnostic accuracy in PPA (Hu et al., 2010c); and others find combination of CSF tau isoform levels and midbrain atrophy improve identification of PSP (Borroni et al., 2010). Multiple modalities of MRI methods, including diffusion-tensor imaging (DTI) of white matter may help identify FTLD patients in dementia cohorts. We have demonstrated increased diagnostic sensitivity to differentiate $\mathrm{AD}$ from FTLD cases using a combination of gray matter (GM) density and DTI measures (McMillan et al., 2012a). In addition, we have also discovered promising diagnostic utility for differentiating FTLD-tau and FTLD-TDP using DTI (unpublished data). Cortical atrophy and CSF biomarker levels appear to be highly correlated as we have recently demonstrated that GM density could predict CSF t-tau and $A \beta_{1-42}$ levels, and these predicted values could accurately distinguish $\mathrm{AD}$ and FTLD (McMillan et al., 2013). These results indicate that MRI could potentially serve as a surrogate for CSF, which would have significant utility for patients where lumbar puncture would be difficult or for clinical trial endpoints where repeated lumbar puncture may be needed. Finally, recent genome-wide association studies (GWAS) have found risk alleles associated with FTLD-TDP (Van Deerlin et al., 2010) and FTLD-tau (Hoglinger et al., 2011). Further knowledge of clinical, neuroimaging, and biofluid correlates of these risk alleles in FTLD could provide further useful diagnostic and prognostic information. Thus, comparative studies of clinical, genetic, biofluid, and neuroimaging biomarkers in longitudinally followed, well-annotated, autopsy-confirmed subjects will be a powerful method for improving our understanding of the pathophysiology of FTLD and further directing diagnostic and treatment efforts.

\section{SUMMARY}

CSF measurements of $A \beta_{1-42}$, t-tau, and $\mathrm{p}$-tau in FTLD differ significantly from the abnormal levels seen in $\mathrm{AD}$, and in a subset of both FTLD-tau and FTLD-TDP there are extremely low levels of t-tau of unclear etiology. These properties allow for accurate distinction of FTLD from AD in autopsy-confirmed cohorts, while FTLD-specific markers are still lacking.

As we move toward therapies that impact the progression of the disease and target the underlying pathophysiology in FTLD and other neurodegenerative disorders it will be essential for clinicians to view these disorders as clinicopathological entities with the underlying neuropathological substrate in mind. Indeed, new clinical criteria for $\mathrm{AD}$ incorporate this ideology with the designation of "pre-symptomatic AD" (Sperling et al., 2011). In the 
study of the complex clinicopathological spectrum of FTLD disorders, where heterogeneity is the rule, useful markers to develop homogenous clinical, genetic, and neuropathologic subgroups will be crucial to further our goals toward meaningful treatments that could potential slow disease progression and limit patient disability.

\section{REFERENCES}

Alladi, S., Xuereb, J., Bak, T., Nestor, P., Knibb, J., Patterson, K., et al. (2007). Focal cortical presentations of Alzheimer's disease. Brain 130, 2636-2645.

Arai, H., Morikawa, Y., Higuchi, M., Matsui, T., Clark, C. M., Miura, M., et al. (1997). Cerebrospinal fluid tau levels in neurodegenerative diseases with distinct tau-related pathology. Biochem. Biophys. Res. Commun. 236, 262-264.

Ash, S., Moore, P., Antani, S., McCawley, G., Work, M., and Grossman, M. (2006). Trying to tell a tale: discourse impairments in progressive aphasia and frontotemporal dementia. Neurology 66, 1405-1413.

Ash, S., Moore, P., Vesely, L., Gunawardena, D., McMillan, C., Anderson, C., et al. (2009). Non-Fluent Speech in Frontotemporal Lobar Degeneration. J. Neurolinguistics 22, 370-383.

Baker, M., Mackenzie, I. R., PickeringBrown, S. M., Gass, J., Rademakers, R., Lindholm, C., et al. (2006). Mutations in progranulin cause taunegative frontotemporal dementia linked to chromosome 17. Nature 442, 916-919.

Bateman, R. J., Xiong, C., Benzinger, T. L., Fagan, A. M., Goate, A., Fox, N. C., et al. (2012). Clinical and biomarker changes in dominantly inherited Alzheimer's disease. N. Engl. J. Med. 367, 795-804.

Bian, H., and Grossman, M. (2007). Frontotemporal lobar degeneration: recent progress in antemortem diagnosis. Acta Neuropathol. 114, 23-29.

Bian, H., Van Swieten, J. C., Leight, S., Massimo, L., Wood, E., Forman, M., et al. (2008). CSF biomarkers in frontotemporal lobar degeneration with known pathology. Neurology 70, 1827-1835.

Bibl, M., Gallus, M., Welge, V., Esselmann, H., Wolf, S., Ruther, E., et al. (2012). Cerebrospinal fluid amyloid-beta 2-42 is decreased in Alzheimer's, but not in frontotemporal dementia. J. Neural Transm. 119, 805-813.

Bibl, M., Mollenhauer, B., Lewczuk, P., Esselmann, H., Wolf, S., Otto,
M., et al. (2011). Cerebrospinal fluid tau, p-tau 181 and amyloidbeta38/40/42 in frontotemporal dementias and primary progressive aphasias. Dement. Geriatr. Cogn. Disord. 31, 37-44.

Bibl, M., Mollenhauer, B., Wolf, S., Esselmann, H., Lewczuk, P., Kornhuber, J., et al. (2007). Reduced CSF carboxyterminally truncated Abeta peptides in frontotemporal lobe degenerations.

Blennow, K., Wallin, A., Agren, H., Spenger, C., Siegfried, J., and Vanmechelen, E. (1995). Tau protein in cerebrospinal fluid: a biochemical marker for axonal degeneration in Alzheimer disease? Mol. Chem. Neuropathol. 26, 231-245.

Borroni, B., Gardoni, F., Parnetti, L., Magno, L., Malinverno, M., Saggese, E., et al. (2009). Pattern of Tau forms in CSF is altered in progressive supranuclear palsy. Neurobiol. Aging 30, 34-40.

Borroni, B., Malinverno, M., Gardoni, F., Alberici, A., Parnetti, L., Premi, E., et al. (2008). Tau forms in CSF as a reliable biomarker for progressive supranuclear palsy. Neurology 71, 1796-1803.

Borroni, B., Malinverno, M., Gardoni, F., Grassi, M., Parnetti, L., Agosti, C., et al. (2010). A combination of CSF tau ratio and midsaggital midbrain-to-pons atrophy for the early diagnosis of progressive supranuclear palsy. J. Alzheimers Dis. 22, 195-203.

Boxer, A. L., Gold, M., Huey, E., Gao, F. B., Burton, E. A., Chow, T., et al. (2012a). Frontotemporal degeneration, the next therapeutic frontier: Molecules and animal models for frontotemporal degeneration drug development. Alzheimers Dement. doi: 10.1016/j.jalz.2012.03. 002. [Epub ahead of print].

Boxer, A. L., Gold, M., Huey, E., $\mathrm{Hu}$, W. T., Rosen, H., Kramer, J., et al. (2012b). The advantages of frontotemporal degeneration drug development (part 2 of frontotemporal degeneration: the next therapeutic frontier). Alzheimers Dement. doi: 10.1016/j.jalz.2012.03. 003. [Epub ahead of print]. J. Neural Transm. 114, 621-628.

\section{ACKNOWLEDGMENTS}

We thank the patients studied here and their families who made the research reviewed here possible. Funding for this study was provided by the National Institutes of Health grants P30 AG10124, AG17586, and T32-AG000255 as well as the Wyncote Foundation.

Brunnstrom, H., Rawshani, N. Zetterberg, H., Blennow, K. Minthon, L., Passant, U., et al. (2010). Cerebrospinal fluid biomarker results in relation to neuropathological dementia diagnoses. Alzheimers Dement. 6 , 104-109.

Buerger, K., Teipel, S. J., Zinkowski, R., Blennow, K., Arai, H., Engel, R., et al. (2002a). CSF tau protein phosphorylated at threonine 231 correlates with cognitive decline in MCI subjects. Neurology 59, 627-629.

Buerger, K., Zinkowski, R., Teipel, S. J., Tapiola, T., Arai, H., Blennow, K., et al. (2002b). Differential diagnosis of Alzheimer disease with cerebrospinal fluid levels of tau protein phosphorylated at threonine 231 . Arch. Neurol. 59, 1267-1272.

Clark, C. M., Xie, S., Chittams, J., Ewbank, D., Peskind, E., Galasko, D., et al. (2003). Cerebrospinal fluid tau and beta-amyloid: how well do these biomarkers reflect autopsy-confirmed dementia diagnoses? Arch. Neurol. 60, 1696-1702.

Cohen, T. J., Guo, J. L., Hurtado, D. E., Kwong, L. K., Mills, I. P. Trojanowski, J. Q., et al. (2011). The acetylation of tau inhibits its function and promotes pathological tau aggregation. Nat. Commun. 2:252 doi: 10.1038/ncomms1255

Cruts, M., Gijselinck, I., Van Der Zee, J., Engelborghs, S., Wils, H., Pirici, D., et al. (2006). Null mutations in progranulin cause ubiquitin-positive frontotemporal dementia linked to chromosome 17q21. Nature 442, 920-924.

Davis, D. G., Schmitt, F. A., Wekstein, D. R., and Markesbery, W. R. (1999). Alzheimer neuropathologic alterations in aged cognitively normal subjects. J. Neuropathol. Exp. Neurol. 58, 376-388.

De Jong, D., Jansen, R. W., Pijnenburg, Y. A., Van Geel, W. J., Borm, G. F., Kremer, H. P., et al. (2007). CSF neurofilament proteins in the differential diagnosis of dementia. J. Neurol. Neurosurg. Psychiatry 78, 936-938.

De Meyer, G., Shapiro, F., Vanderstichele, H., Vanmechelen,
E., Engelborghs, S., De Deyn, P. P., et al. (2010). Diagnosisindependent Alzheimer disease biomarker signature in cognitively normal elderly people. Arch. Neurol. 67, 949-956.

De Souza, L. C., Lamari, F., Belliard, S., Jardel, C., Houillier, C., De Paz, R., et al. (2011). Cerebrospinal fluid biomarkers in the differential diagnosis of Alzheimer's disease from other cortical dementias. J. Neurol. Neurosurg. Psychiatry 82, 240-246.

Dejesus-Hernandez, M., Mackenzie, I. R., Boeve, B. F., Boxer, A. L., Baker, M., Rutherford, N. J., et al. (2011). Expanded GGGGCC hexanucleotide repeat in noncoding region of C9ORF72 causes chromosome 9p-linked FTD and ALS. Neuron 72, 245-256.

Dickson, D. (2004). "Sporadic tauopaties: Pick's disease, corticobasal degeneration, progressive supranuclear palsy and argyrophilic grain disease," in The Neuropathology of Dementia 2nd $E d n$, eds M. Esiri, V. M-Y. Lee, and J. Q. Trojanowski (New York, NY: Cambridge University Press), 227-256.

Engelborghs, S., De Vreese, K., Van De Casteele, T., Vanderstichele, H., Van Everbroeck, B., Cras, P., et al. (2008). Diagnostic performance of a CSF-biomarker panel in autopsyconfirmed dementia. Neurobiol. Aging 29, 1143-1159.

Engelborghs, S., Maertens, K., Vloeberghs, E., Aerts, T. Somers, N., Marien, P., et al. (2006). Neuropsychological and behavioural correlates of CSF biomarkers in dementia. Neurochem. Int. 48, 286-295.

Eslinger, P. J., Moore, P., Antani, S., Anderson, C., and Grossman, M. (2012). Apathy in frontotemporal dementia: behavioral and neuroimaging correlates. Behav. Neurol. 25, 127-136

Fagan, A. M., Mintun, M. A., Mach, R. H., Lee, S. Y., Dence, C. S., Shah, A. R., et al. (2006). Inverse relation between in vivo amyloid imaging load and cerebrospinal fluid Abeta42 in humans. Ann. Neurol. 59, 512-519. 
Fagan, A. M., Shaw, L. M., Xiong, C., Vanderstichele, H., Mintun, M. A., Trojanowski, J. Q., et al. (2011). Comparison of analytical platforms for cerebrospinal fluid measures of \{beta\}-amyloid 1-42, total tau, and P-taul81 for identifying alzheimer disease amyloid plaque pathology. Arch. Neurol. 68, 1137-1144.

Forman, M. S., Farmer, J., Johnson, J. K., Clark, C. M., Arnold, S. E., Coslett, H. B., et al. (2006). Frontotemporal dementia: clinicopathological correlations. Ann. Neurol. 59, 952-962.

Foulds, P., McAuley, E., Gibbons, L., Davidson, Y., Pickering-Brown, S. M., Neary, D., et al. (2008). TDP-43 protein in plasma may index TDP43 brain pathology in Alzheimer's disease and frontotemporal lobar degeneration. Acta Neuropathol. 116, 141-146.

Gabelle, A., Roche, S., Geny, C., Bennys, K., Labauge, P., Tholance, Y., et al. (2011). Decreased sAbetaPPbeta, Abeta38, and Abeta40 cerebrospinal fluid levels in frontotemporal dementia. J. Alzheimers Dis. 26, 553-563.

Geser, F., Brandmeir, N. J., Kwong, L. K., Martinez-Lage, M., Elman, L., McCluskey, L., et al. (2008). Evidence of multisystem disorder in whole-brain map of pathological TDP-43 in amyotrophic lateral sclerosis. Arch. Neurol. 65, 636-641.

Geser, F., Martinez-Lage, M., Robinson, J., Uryu, K., Neumann, M., Brandmeir, N. J., et al. (2009). Clinical and pathological continuum of multisystem TDP-43 proteinopathies. Arch. Neurol. 66, 180-189.

Ghidoni, R., Benussi, L., Glionna, M., Franzoni, M., and Binetti, G. (2008). Low plasma progranulin levels predict progranulin mutations in frontotemporal lobar degeneration. Neurology 71, 1235-1239.

Gorno-Tempini, M. L., Brambati, S. M., Ginex, V., Ogar, J., Dronkers, N. F., Marcone, A., et al. (2008). The logopenic/phonological variant of primary progressive aphasia. Neurology 71, 1227-1234.

Gorno-Tempini, M. L., Dronkers, N. F., Rankin, K. P., Ogar, J. M., Phengrasamy, L., Rosen, H. J., et al. (2004). Cognition and anatomy in three variants of primary progressive aphasia. Ann. Neurol. 55, 335-346.

Gorno-Tempini, M. L., Hillis, A. E., Weintraub, S., Kertesz, A., Mendez, M., Cappa, S. F., et al. (2011). Classification of primary progressive aphasia and its variants. Neurology 76, 1006-1014.

Green, A. J., Harvey, R. J., Thompson, E. J., and Rossor, M. N. (1999). Increased tau in the cerebrospinal fluid of patients with frontotemporal dementia and Alzheimer's disease. Neurosci. Lett. 259, 133-135.

Grossman, M. (2010). Primary progressive aphasia: clinicopathological correlations. Nat. Rev. Neurol. 6, 88-97.

Grossman, M., Eslinger, P. J., Troiani, V., Anderson, C., Avants, B., Gee, J. C., et al. (2010). The role of ventral medial prefrontal cortex in social decisions: converging evidence from fMRI and frontotemporal lobar degeneration. Neuropsychologia 48, 3505-3512.

Grossman, M., Farmer, J., Leight, S., Work, M., Moore, P., Van Deerlin, V., et al. (2005). Cerebrospinal fluid profile in frontotemporal dementia and Alzheimer's disease. Ann. Neurol. 57, 721-729.

Grossman, M., Powers, J., Ash, S., McMillan, C., Burkholder, L., Irwin, D., et al. (2012). Disruption of large-scale neural networks in non-fluent/agrammatic variant primary progressive aphasia associated with frontotemporal degeneration pathology. Brain Lang. doi: 10.1016/ j.bandl.2012.10.005. [Epub ahead of print].

Grossman, M., Xie, S. X., Libon, D. J., Wang, X., Massimo, L., Moore, P., et al. (2008). Longitudinal decline in autopsy-defined frontotemporal lobar degeneration. Neurology 70, 2036-2045.

Gunawardena, D., Ash, S., McMillan, C., Avants, B., Gee, J., and Grossman, M. (2010). Why are patients with progressive nonfluent aphasia nonfluent? Neurology 75, 588-594.

Hampel, H., Blennow, K., Shaw, L. M., Hoessler, Y. C., Zetterberg, H., and Trojanowski, J. Q. (2010). Total and phosphorylated tau protein as biological markers of Alzheimer's disease. Exp. Gerontol. 45, 30-40.

Hesse, C., Rosengren, L., Vanmechelen, E., Vanderstichele, H., Jensen, C., Davidsson, P., et al. (2000). Cerebrospinal fluid markers for Alzheimer's disease evaluated after acute ischemic stroke. J. Alzheimers Dis. 2, 199-206.

Hodges, J. R., Davies, R. R., Xuereb, J. H., Casey, B., Broe, M., Bak, T. H., et al. (2004). Clinicopathological correlates in frontotemporal dementia. Ann. Neurol. 56, 399-406.

Hodges, J. R., and Patterson, K. (2007). Semantic dementia: a unique clinicopathological syndrome. Lancet Neurol. 6, 1004-1014.

Hoglinger, G. U., Melhem, N. M. Dickson, D. W., Sleiman, P. M. Wang, L. S., Klei, L., et al. (2011) Identification of common variants influencing risk of the tauopathy progressive supranuclear palsy. Nat. Genet. 43, 699-705.

$\mathrm{Hu}$, W. T., Chen-Plotkin, A., Arnold, S. E., Grossman, M., Clark, C. M., Shaw, L. M., et al. (2010a). Biomarker discovery for Alzheimer's disease, frontotemporal lobar degeneration, and Parkinson's disease. Acta Neuropathol. 120, 385-399.

Hu, W. T., Chen-Plotkin, A., Grossman, M., Arnold, S. E., Clark, C. M., Shaw, L. M., et al. (2010b). Novel CSF biomarkers for frontotemporal lobar degenerations. Neurology 75, 2079-2086.

Hu, W. T., McMillan, C., Libon, D. Leight, S., Forman, M., Lee, V. M. et al. (2010c). Multimodal predictors for Alzheimer disease in nonfluent primary progressive aphasia. Neurology 75, 595-602.

Hu, W. T., Trojanowski, J. Q. and Shaw, L. M. (2011). Biomarkers in frontotemporal lobar degenerations-progress and challenges. Prog. Neurobiol. 95, 636-648.

Hutton, M., Lendon, C. L., Rizzu, P., Baker, M., Froelich, S., Houlden, H., et al. (1998). Association of missense and 5'-splice-site mutations in tau with the inherited dementia FTDP-17. Nature 393 702-705.

Irwin, D. J., Cohen, T. J., Grossman, M., Arnold, S. E., Xie, S. X., Lee, V. M., et al. (2012a). Acetylated tau, a novel pathological signature in Alzheimer's disease and other tauopathies. Brain 135, 807-818.

Irwin, D. J., McMillan, C. T., Toledo, J. B., Arnold, S. E., Shaw, L. M., Wang, L. S., et al. (2012b). Comparison of cerebrospinal fluid levels of tau and Abeta 1-42 in Alzheimer disease and frontotemporal degeneration using 2 analytical platforms. Arch. Neurol. 69, 1018-1025.

Irwin, D. J., McMillan, C. T., Brettschneider, J., Libon, D. J., Powers, J., Rascovsky, K., et al. (2013). Cognitive decline and reduced survival in C9orf72 expansion frontotemporal degeneration and amyotrophic lateral sclerosis. J. Neurol. Neurosurg. Psychiatry. 84, 163-169.

Jack, C. R. Jr., Knopman, D. S., Jagust, W. J., Shaw, L. M., Aisen, P. S., Weiner, M. W., et al. (2010).
Hypothetical model of dynamic biomarkers of the Alzheimer's pathological cascade. Lancet Neurol. 9, 119-128.

Jack, C. R. Jr., Vemuri, P., Wiste, H. J., Weigand, S. D., Lesnick, T. G., Lowe, V., et al. (2012). Shapes of the trajectories of 5 major biomarkers of Alzheimer disease. Arch. Neurol. 69, 856-867.

Jin, K., Takeda, A., Shiga, Y., Sato, S., Ohnuma, A., Nomura, H., et al. (2006). CSF tau protein: a new prognostic marker for Guillain-Barre syndrome. Neurology 67, 1470-1472.

Josephs, K. A. (2008). Frontotemporal dementia and related disorders: deciphering the enigma. Ann. Neurol. 64, 4-14.

Josephs, K. A., Duffy, J. R., Strand, E. A., Whitwell, J. L., Layton, K. F., Parisi, J. E., et al. (2006a). Clinicopathological and imaging correlates of progressive aphasia and apraxia of speech. Brain 129, 1385-1398.

Josephs, K. A., Whitwell, J. L., Jack, C. R., Parisi, J. E., and Dickson, D. W. (2006b). Frontotemporal lobar degeneration without lobar atrophy. Arch. Neurol. 63, 1632-1638.

Kapaki, E., Paraskevas, G. P., Papageorgiou, S. G., Bonakis, A., Kalfakis, N., Zalonis, I., et al. (2008). Diagnostic value of CSF biomarker profile in frontotemporal lobar degeneration. Alzheimer Dis. Assoc. Disord. 22, 47-53.

Kasai, T., Tokuda, T., Ishigami, N., Sasayama, H., Foulds, P., Mitchell, D. J., et al. (2009). Increased TDP43 protein in cerebrospinal fluid of patients with amyotrophic lateral sclerosis. Acta Neuropathol. 117, 55-62.

Kertesz, A., McMonagle, P., Blair, M., Davidson, W., and Munoz, D. G. (2005). The evolution and pathology of frontotemporal dementia. Brain 128, 1996-2005.

Kipps, C. M., Hodges, J. R. and Hornberger, M. (2010). Nonprogressive behavioural frontotemporal dementia: recent developments and clinical implications of the 'bvFTD phenocopy syndrome'. Curr. Opin. Neurol. 23, 628-632.

Knibb, J. A., Xuereb, J. H., Patterson, K., and Hodges, J. R. (2006). Clinical and pathological characterization of progressive aphasia. Ann. Neurol. 59, 156-165.

Knopman, D. S., Boeve, B. F., Parisi, J. E., Dickson, D. W., Smith, G. E., Ivnik, R. J., et al. (2005). Antemortem diagnosis of 
frontotemporal lobar degeneration. Ann. Neurol. 57, 480-488.

Knopman, D. S., Kramer, J. H., Boeve, B. F., Caselli, R. J., Graff-Radford, N. R., Mendez, M. F., et al. (2008). Development of methodology for conducting clinical trials in frontotemporal lobar degeneration. Brain 131, 2957-2968.

Koedam, E. L., Van Der Vlies, A. E., Van Der Flier, W. M., Verwey, N. A., Koene, T., Scheltens, P., et al. (2012). Cognitive correlates of cerebrospinal fluid biomarkers in frontotemporal dementia. Alzheimers Dement. doi: 10.1016/j.jalz.2011.12. 007. [Epub ahead of print].

Koopman, K., Le Bastard, N., Martin, J. J., Nagels, G., De Deyn, P. P., and Engelborghs, S. (2009). Improved discrimination of autopsy-confirmed Alzheimer's disease $(\mathrm{AD})$ from non-AD dementias using CSF P-tau(181P). Neurochem. Int. 55, 214-218.

Koric, L., Felician, O., Guedj, E., Hubert, A. M., Mancini, J., Boucraut, J., et al. (2010). Could clinical profile influence CSF biomarkers in earlyonset Alzheimer disease? Alzheimer Dis. Assoc. Disord. 24, 278-283.

Krut, J. J., Zetterberg, H., Blennow, K., Cinque, P., Hagberg, L., Price, R. W., et al. (2013). Cerebrospinal fluid Alzheimer's biomarker profiles in CNS infections. J. Neurol. 260, 620-626.

Lee, S. E., Rabinovici, G. D., Mayo, M. C., Wilson, S. M., Seeley, W. W., Dearmond, S. J., et al. (2011). Clinicopathological correlations in corticobasal degeneration. Ann. Neurol. 70, 327-340.

Lewczuk, P., Zimmermann, R., Wiltfang, J., and Kornhuber, J. (2009). Neurochemical dementia diagnostics: a simple algorithm for interpretation of the CSF biomarkers. J Neural Transm 116, 1163-1167.

Libon, D. J., Rascovsky, K., Gross, R. G., White, M. T., Xie, S. X., Dreyfuss, M., et al. (2011). The Philadelphia Brief Assessment of Cognition (PBAC): a validated screening measure for dementia. Clin. Neuropsychol. 25, 1314-1330.

Luk, C., Compta, Y., Magdalinou, N., Marti, M. J., Hondhamuni, G., Zetterberg, H., et al. (2012). Development and assessment of sensitive immuno-PCR assays for the quantification of cerebrospinal fluid three- and four-repeat tau isoforms in tauopathies. J. Neurochem. 123, 396-405.

Mackenzie, I. R., Neumann, M., Baborie, A., Sampathu, D. M., Du
Plessis, D., Jaros, E., et al. (2011). A harmonized classification system for FTLD-TDP pathology. Acta Neuropathol. 122, 111-113.

Mackenzie, I. R., Neumann, M., Bigio, E. H., Cairns, N. J., Alafuzoff, I., Kril, J., et al. (2010). Nomenclature and nosology for neuropathologic subtypes of frontotemporal lobar degeneration: an update. Acta Neuropathol. 119, 1-4.

Massimo, L., Libon, D. J., Chandrasekaran, K., Dreyfuss, M., McMillan, C. T., Rascovsky, K., et al. (2013). Self-appraisal in behavioural variant frontotemporal degeneration. J. Neurol. Neurosurg. Psychiatry, 84, 148-153.

Massimo, L., Powers, C., Moore, P., Vesely, L., Avants, B., Gee, J., et al. (2009). Neuroanatomy of apathy and disinhibition in frontotemporal lobar degeneration. Dement. Geriatr. Cogn. Disord. 27, 96-104.

Matsuo, E. S., Shin, R. W., Billingsley, M. L., Van Devoorde, A., O'Connor, M., Trojanowski, J. Q., et al. (1994). Biopsy-derived adult human brain tau is phosphorylated at many of the same sites as Alzheimer's disease paired helical filament tau. Neuron 13, 989-1002.

Mattsson, N., Andreasson, U., Persson, S., Arai, H., Batish, S. D., Bernardini, S., et al. (2011). The Alzheimer's association external quality control program for cerebrospinal fluid biomarkers. Alzheimers Dement. 7, 386-395. e386.

Mattsson, N., Ruetschi, U., Pijnenburg, Y. A., Blankenstein, M. A., Podust, V. N., Li, S., et al. (2008). Novel cerebrospinal fluid biomarkers of axonal degeneration in frontotemporal dementia. Mol. Med. Report. 1, 757-761.

McMillan, C. T., Avants, B., Irwin, D. J., Toledo, J. B., Wolk, D. A., Van Deerlin, V. M., et al. (2013). Can MRI screen for CSF biomarkers in neurodegenerative disease? Neurology, 80, 132-138.

McMillan, C. T., Brun, C., Siddiqui, S., Churgin, M., Libon, D., Yushkevich, P., et al. (2012a). White matter imaging contributes to the multimodal diagnosis of frontotemporal lobar degeneration. Neurology 78, 1761-1768.

McMillan, C. T., Rascovsky, K., Khella, M. C., Clark, R., and Grossman, M. (2012b). The neural basis for establishing a focal point in pure coordination games. Soc. Cogn. Affect. Neurosci. 7, 881-887.

Mesulam, M., Wicklund, A., Johnson, N., Rogalski, E., Leger, G. C.,
Rademaker, A., et al. (2008). Alzheimer and frontotemporal pathology in subsets of primary progressive aphasia. Ann. Neurol. 63, 709-719.

Mesulam, M. M. (1982). Slowly progressive aphasia without generalized dementia. Ann. Neurol. 11, 592-598.

Mesulam, M. M. (2001). Primary progressive aphasia. Ann. Neurol. 49, 425-432.

NLM/NIH. 2012. Available online at: http://www.ncbi.nlm.nih.gov/ pubmed/ [Accessed 10/27/12 2012].

Olsson, A., Vanderstichele, H. Andreasen, N., De Meyer, G., Wallin, A., Holmberg, B., et al. (2005). Simultaneous measurement of beta-amyloid(1-42), total tau, and phosphorylated tau (Thr181) in cerebrospinal fluid by the xMAP technology. Clin. Chem. 51, 336-345.

Ost, M., Nylen, K., Csajbok, L., Ohrfelt, A. O., Tullberg, M., Wikkelso, C., et al. (2006). Initial CSF total tau correlates with 1-year outcome in patients with traumatic brain injury. Neurology 67, 1600-1604.

Otto, M., Wiltfang, J., Tumani, H., Zerr, I., Lantsch, M., Kornhuber, J., et al. (1997). Elevated levels of tau-protein in cerebrospinal fluid of patients with Creutzfeldt-Jakob disease. Neurosci. Lett. 225, 210-212.

Patel, S., Lee, E. B., Xie, S. X., Law, A., Jackson, E. M., Arnold, S. E., et al. (2012). Phosphorylated tau/amyloid beta 1-42 ratio in ventricular cerebrospinal fluid reflects outcome in idiopathic normal pressure hydrocephalus. Fluids Barriers CNS 9:7. doi: 10.1186/2045-81189-7

Pijnenburg, Y. A., Schoonenboom, N. S., Rosso, S. M., Mulder, C., Van Kamp, G. J., Van Swieten, J. C., et al. (2004). CSF tau and Abeta42 are not useful in the diagnosis of frontotemporal lobar degeneration. Neurology 62, 1649.

Pijnenburg, Y. A., Schoonenboom, S. N., Mehta, P. D., Mehta, S. P., Mulder, C., Veerhuis, R., et al. (2007). Decreased cerebrospinal fluid amyloid beta (1-40) levels in frontotemporal lobar degeneration. J. Neurol. Neurosurg. Psychiatry 78, 735-737.

Rabinovici, G. D., Jagust, W. J., Furst, A. J., Ogar, J. M., Racine, C. A., Mormino, E. C., et al. (2008). Abeta amyloid and glucose metabolism in three variants of primary progressive aphasia. Ann. Neurol. 64, 388-401.

Rascovsky, K., Hodges, J. R., Knopman, D., Mendez, M. F., Kramer, J. H., Neuhaus, J., et al. (2011). Sensitivity of revised diagnostic criteria for the behavioural variant of frontotemporal dementia. Brain 134, 2456-2477.

Rascovsky, K., Salmon, D. P., Hansen, L. A., and Galasko, D. (2008). Distinct cognitive profiles and rates of decline on the Mattis Dementia Rating Scale in autopsy-confirmed frontotemporal dementia and Alzheimer's disease. J. Int. Neuropsychol. Soc. 14, 373-383.

Renton, A. E., Majounie, E., Waite, A., Simon-Sanchez, J., Rollinson, S., Gibbs, J. R., et al. (2011). A hexanucleotide repeat expansion in C9ORF72 is the cause of chromosome 9p21-linked ALSFTD. Neuron 72, 257-268.

Riemenschneider, M., Wagenpfeil, S., Diehl, J., Lautenschlager, N., Theml, T., Heldmann, B., et al. (2002). Tau and Abeta42 protein in CSF of patients with frontotemporal degeneration. Neurology 58, 1622-1628.

Rosso, S. M., Van Herpen, E., Pijnenburg, Y. A., Schoonenboom, N. S., Scheltens, P., Heutink, P., et al. (2003). Total tau and phosphorylated tau 181 levels in the cerebrospinal fluid of patients with frontotemporal dementia due to P301L and G272V tau mutations. Arch. Neurol. 60, 1209-1213.

Schmidt, M. L., Gur, R. E., Gur, R. C., and Trojanowski, J. Q. (1988). Intraneuronal and extracellular neurofibrillary tangles exhibit mutually exclusive cytoskeletal antigens. Ann. Neurol. 23, 184-189.

Schoonenboom, N. S., Pijnenburg, Y. A., Mulder, C., Rosso, S. M., Van Elk, E. J., Van Kamp, G. J., et al. (2004). Amyloid beta(1-42) and phosphorylated tau in CSF as markers for early-onset Alzheimer disease. Neurology 62, 1580-1584.

Schoonenboom, N. S., Reesink, F. E., Verwey, N. A., Kester, M. I., Teunissen, C. E., Van De Ven, P. M., et al. (2012). Cerebrospinal fluid markers for differential dementia diagnosis in a large memory clinic cohort. Neurology 78, 47-54.

Seguin, J., Formaglio, M., PerretLiaudet, A., Quadrio, I., Tholance, Y., Rouaud, O., et al. (2011). CSF biomarkers in posterior cortical atrophy. Neurology 76, 1782-1788.

Seppala, T. T., Nerg, O., Koivisto, A. M., Rummukainen, J., Puli, L., Zetterberg, H., et al. (2012). CSF biomarkers for Alzheimer disease correlate with cortical brain biopsy findings. Neurology 78, 1568-1575.

Shaw, L. M., Vanderstichele, H., Knapik-Czajka, M., Clark, C. M., Aisen, P. S., Petersen, R. C., 
et al. (2009). Cerebrospinal fluid biomarker signature in Alzheimer's disease neuroimaging initiative subjects. Ann. Neurol. 65, 403-413.

Shaw, L. M., Vanderstichele, H., Knapik-Czajka, M., Figurski, M., Coart, E., Blennow, K., et al. (2011). Qualification of the analytical and clinical performance of CSF biomarker analyses in ADNI. Acta Neuropathol. 121, 597-609.

Shi, J., Shaw, C. L., Du Plessis, D., Richardson, A. M., Bailey, K. L., Julien, C., et al. (2005). Histopathological changes underlying frontotemporal lobar degeneration with clinicopathological correlation. Acta Neuropathol. 110, 501-512.

Sjogren, M., Davidsson, P., Tullberg, M., Minthon, L., Wallin, A., Wikkelso, C., et al. (2001). Both total and phosphorylated tau are increased in Alzheimer's disease. J. Neurol. Neurosurg. Psychiatry 70, 624-630.

Sjogren, M., Minthon, L., Davidsson, P., Granerus, A. K., Clarberg, A., Vanderstichele, H., et al. (2000a). CSF levels of tau, betaamyloid(1-42) and GAP-43 in frontotemporal dementia, other types of dementia and normal aging. J. Neural Transm. 107, 563-579.

Sjogren, M., Rosengren, L., Minthon, L., Davidsson, P., Blennow, K., and Wallin, A. (2000b). Cytoskeleton proteins in CSF distinguish frontotemporal dementia from $\mathrm{AD}$. Neurology 54, 1960-1964.

Snowden, J., Neary, D., and Mann, D. (2007). Frontotemporal lobar degeneration: clinical and pathological relationships. Acta Neuropathol. 114, 31-38.

Sperling, R. A., Aisen, P. S., Beckett, L. A., Bennett, D. A., Craft, S., Fagan, A. M., et al. (2011). Toward defining the preclinical stages of
Alzheimer's disease: recommendations from the National Institute on Aging-Alzheimer's Association workgroups on diagnostic guidelines for Alzheimer's disease. Alzheimers Dement. 7, 280-292.

Steinacker, P., Hendrich, C., Sperfeld, A. D., Jesse, S., Von Arnim, C. A., Lehnert, S., et al. (2008). TDP-43 in cerebrospinal fluid of patients with frontotemporal lobar degeneration and amyotrophic lateral sclerosis. Arch. Neurol. 65, 1481-1487.

Tapiola, T., Alafuzoff, I., Herukka, S. K., Parkkinen, L., Hartikainen, P., Soininen, H., et al. (2009). Cerebrospinal fluid $\{$ beta $\}$-amyloid 42 and tau proteins as biomarkers of Alzheimer-type pathologic changes in the brain. Arch. Neurol. $66,382-389$.

Toledo, J. B., Brettschneider, J., Grossman, M., Arnold, S. E., Hu, W. T., Xie, S. X., et al. (2012). CSF biomarkers cutoffs: the importance of coincident neuropathological diseases. Acta Neuropathol. 124, 23-35.

Trojanowski, J. Q., and Growdon, J. H. (1998). A new consensus report on biomarkers for the early antemortem diagnosis of Alzheimer disease: current status, relevance to drug discovery, and recommendations for future research. J. Neuropathol. Exp. Neurol. 57, 643-644.

Trojanowski, J. Q., Vandeerstichele, H., Korecka, M., Clark, C. M., Aisen, P. S., Petersen, R. C., et al. (2010). Update on the biomarker core of the Alzheimer's Disease Neuroimaging Initiative subjects. Alzheimers Dement. 6, 230-238.

Turner, R. S., Kenyon, L. C., Trojanowski, J. Q., Gonatas, N., and Grossman, M. (1996). Clinical, neuroimaging, and pathologic features of progressive nonfluent aphasia. Ann. Neurol. 39, 166-173.
Van Deerlin, V. M., Sleiman, P. M., Martinez-Lage, M., Chen-Plotkin, A., Wang, L. S., Graff-Radford, N. R., et al. (2010). Common variants at $7 \mathrm{p} 21$ are associated with frontotemporal lobar degeneration with TDP-43 inclusions. Nat. Genet. 42, 234-239.

Van Harten, A. C., Kester, M. I., Visser, P. J., Blankenstein, M. A., Pijnenburg, Y. A., Van Der Flier, W. M., et al. (2011). Tau and p-tau as CSF biomarkers in dementia: a meta-analysis. Clin. Chem. Lab. Med. 49, 353-366.

Vanmechelen, E., Vanderstichele, H., Davidsson, P., Van Kerschaver, E., Van Der Perre, B., Sjogren, M., et al. (2000). Quantification of tau phosphorylated at threonine 181 in human cerebrospinal fluid: a sandwich ELISA with a synthetic phosphopeptide for standardization. Neurosci. Lett. 285, 49-52.

Verwey, N. A., Kester, M. I., Van Der Flier, W. M., Veerhuis, R., Berkhof, H., Twaalfhoven, H., et al. (2010). Additional value of CSF amyloid-beta 40 levels in the differentiation between FTLD and control subjects. J. Alzheimers Dis. 20, 445-452.

Wang, L. S., Leung, Y. Y., Chang, S. K., Leight, S., Knapik-Czajka, M., Baek, Y., et al. (2012). Comparison of xMAP and ELISA assays for detecting cerebrospinal fluid biomarkers of Alzheimer's disease. J. Alzheimers Dis. 31, 439-445.

Weiner, M. W., Aisen, P. S., Jack, C. R. Jr., Jagust, W. J., Trojanowski, J. Q., Shaw, L., et al. (2010). The Alzheimer's disease neuroimaging initiative: progress report and future plans. Alzheimers Dement. 6, 202211 e207.

Whitwell, J. L., Weigand, S. D., Boeve, B. F., Senjem, M. L., Gunter, J. L., Dejesus-Hernandez, M., et al. (2012). Neuroimaging signatures of frontotemporal dementia genetics: C9ORF72, tau, progranulin and sporadics. Brain 135, 794-806.

Zhukareva, V., Sundarraj, S., Mann, D. Sjogren, M., Blenow, K., Clark, C. M., et al. (2003). Selective reduction of soluble tau proteins in sporadic and familial frontotemporal dementias: an international followup study. Acta Neuropathol. 105, 469-476.

Zhukareva, V., Vogelsberg-Ragaglia, V., Van Deerlin, V. M., Bruce, J., Shuck, T., Grossman, M., et al. (2001). Loss of brain tau defines novel sporadic and familial tauopathies with frontotemporal dementia. Ann. Neurol. 49, 165-175.

Conflict of Interest Statement: Dr. John Q. Trojanowski reports single consulting services to Pfizer, J\&J, MetLife, and BMS; Royalty payments through Penn licenses; and research support from AstraZeneca and BMS. The other authors declare that the research was conducted in the absence of any commercial or financial relationships that could be construed as a potential conflict of interest.

Received: 06 January 2013; paper pending published: 30 January 2013; accepted: 05 February 2013; published online: 21 February 2013.

Citation: Irwin DJ, Trojanowski JQ and Grossman M (2013) Cerebrospinal fluid biomarkers for differentiation of frontotemporal lobar degeneration from Alzheimer's disease. Front. Aging Neurosci. 5:6. doi: 10.3389/fnagi. 2013.00006

Copyright (c) 2013 Irwin, Trojanowski and Grossman. This is an open-access article distributed under the terms of the Creative Commons Attribution License, which permits use, distribution and reproduction in other forums, provided the original authors and source are credited and subject to any copyright notices concerning any third-party graphics etc. 